\title{
Robot Navigation Behaviors Based on Omnidirectional Vision and Information Theory
}

\author{
Boyan Bonev, Miguel Cazorla and Francisco Escolano
}

\begin{abstract}
In this work we present a reactive autonomous robot navigation system based only on omnidirectional vision. It does not rely on any prior knowledge about the environment apart from assuming a structured one, like indoor corridors or outdoor avenues. The direction of the corridor is estimated from the entropy analysis of a 1-D omnidirectional image. The 2-D omnidirectional image is analyzed for obstacle avoidance and for keeping a safety distance from the borders of the corridor. Both methods are non-metric and no 3-D information is needed. The system performs well with different resolutions and the catadioptric sensor needs no calibration. We present results from indoor and outdoor experiments.
\end{abstract}

Index Terms-omnidirectional vision, autonomous robot navigation, entropy

\section{INTRODUCTION}

A UTONOMOUS navigation in a structured environment without any prior knowledge is a crucial ability for mobile robots. Vision-based navigation systems have the advantage of not needing any additional hardware to be installed on the robot. The installation of a range sensor on a mobile robot has an additional cost, while a vision sensor provides more abundant and richer information. Catadioptric sensors provide the robot with a $360^{\circ}$ field of vision, information which can be exploited in different ways for navigation.

Some approaches are oriented toward extracting 3D information, for example the structure from motion approach [3]. This requires the catadioptric sensor to be calibrated. Catadioptric projection can also be modeled by a spherical projection [6], providing a mathematical framework for working with omnidirectional vision.

Other approaches to navigation do not need any 3-D information about the structure of the target [12][13]. In [5] the original 2-D image is transformed into a 2-D orthographic image (Fig. 1.b).

Finally, the 1-D omnidirectional images are simpler, while keeping the benefits of the $360^{\circ}$ field of vision. In [4] these images are used for storing iconically the spatio-temporal route and then using it for navigation. A different approach is to use the information of 1-D images in a reactive way. Inspired in Cataglyphis ants, [2] implements a skylight compass and also detects landmarks on the 1-D images for implementing a homing strategy. Other works use a sequence of these images

Boyan Bonev, Miguel Cazorla and Francisco Escolano are with the University of Alicante.

Dept. de Ciencias de la Computación e Inteligencia Artificial, Universidad de Alicante

Apdo. 99, E-03080 Alicante, Spain

E-mail: boyan@dccia.ua.es a)

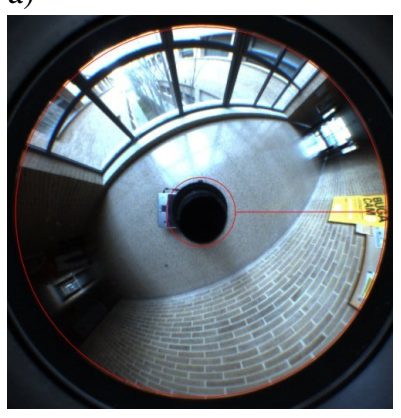

b)

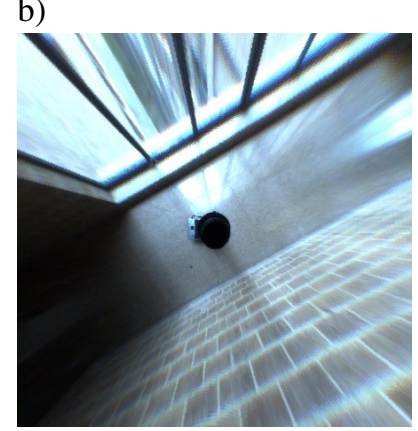

c)

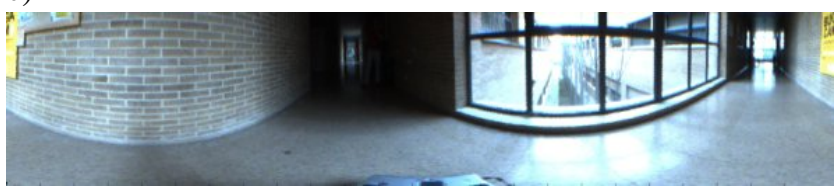

Fig. 1. a) Omnidirectional image obtained with a camera and a hyperbolic mirror. b) Transformation to bird's view (orthographic image). c) Rectified panorama image.

to detect features that are persistent in time and use them for matching and navigation [1].

In the current work we have developed two basic behaviors whose combination allows a robot to navigate along a variety of corridors and avenues. The first behavior estimates the direction of the corridor and makes the robot follow it. The direction is estimated by looking for high entropy zones in the 1-D omnidirectional image. The second behavior is intended to avoid obstacles and uses the original 2-D image. Significant changes in the image are detected as obstacles [7], and the behavior is inspired in sonars ring navigation algorithms [10] [9] [11].Both techniques are not constrained to any particular corridor, as entropy and gradient calculations are applicable to different lighting conditions and any colors. The omnidirectional camera does not need any calibration. It is oriented vertically so that the floor is reflected in the mirror, as shown in Fig. 5.

The rest of the paper is organized as follows. In Section II we detail our entropy-based direction estimation method. Then in Section III we explain the OV-sonars method for visual obstacle avoidance, and in Section IV we propose the combination of both methods for robot navigation. Further on, in Section $\mathrm{V}$ we describe the performed experiments and finally we state our conclusions and further work in Section VI. 


\section{ENTROPY-BASED DIRECTION ESTIMATION}

The estimated direction of the corridor is used for guiding the trajectory of the robot. This estimation is performed in three low-computational-cost steps. First a 1-D omnidirectional image is obtained and geometrically transformed. Then its entropy map is calculated. Finally a low pass filter is applied to the entropy values, in order to find two main peaks in the function.

\section{A. 1-D omnidirectional images}

Our approach intends to find the direction of the corridor using the information of a single shot of the omnidirectional camera. The 1-D omnidirectional image is a row $\vec{l}=\left\{l_{0}, \cdots, l_{w-1}\right\}$ of $w$ pixels $l_{i}$, with a correspondence between $[0, w-1]$ and $[0,360[$.

In [1] the 1-D panorama is obtained from the central row of the rectified image. Our idea is to seize dense features which will vary softly from one image to another. For this reason we perform an average between all the rows of the rectified image:

$$
l_{i}=\frac{1}{h} \sum_{j=1}^{h} I_{R}(i, j)
$$

where $I_{R}$ is the rectified image (Fig. 1.c) with $(w, h)$ width and height.

The sequence of 1-D omnidirectional frames we obtain is represented in Fig. 4. The sequence corresponds to a nonstraight trajectory along a corridor. The final image is a representation of the stack of many consecutive 1-D omnidirectional images. We can see that the changes are soft, and the features on the image are quite dense. We can also observe that both ends of the corridor correspond to the zones in the image with higher density of features.

\section{B. Entropy map}

In a corridor there usually are different obstacles: doors, windows, walls, and several other objects. In the case of outdoors, there are buildings, trees, benches, etc. All of them are features on the image and many of them are covered in a single omnidirectional view. In the ideal case these features will be distributed homogeneously in the corridor, like in Fig. 3. If so, the features will not be homogeneously reflected on the omnidirectional mirror: those which are closer to the mirror will appear more separated from each other, while those that are farther will appear more densely on the image (see a real data example in Fig. 2).

On the other hand, the vertical field of vision of the camera grows with the distance. This means that distant parts of the environment will present many more features than parts which are closer to the camera and are not entirely covered by the field of vision. For a graphical example see Fig. 5.

In real data the features have different colors and intensities. In most cases, the higher the density of features, the higher the local entropy [14]. The entropy map $\vec{h}=\left\{h_{1}, \cdots, h_{w}\right\}$ of

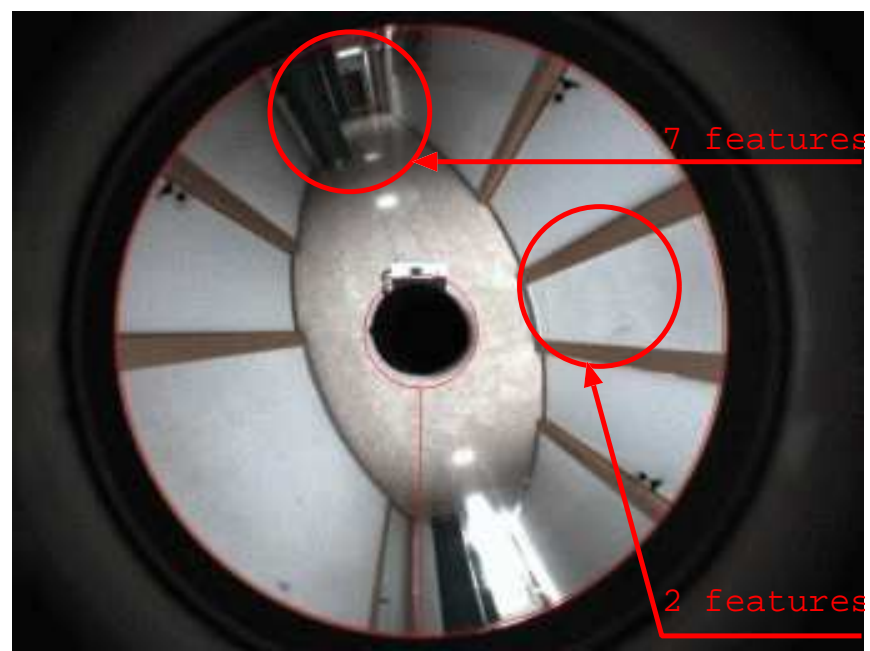

Fig. 2. A sample omnidirectional image taken in a corridor. The density of features is higher in the areas of the image corresponding to more distant obstacles.

a)

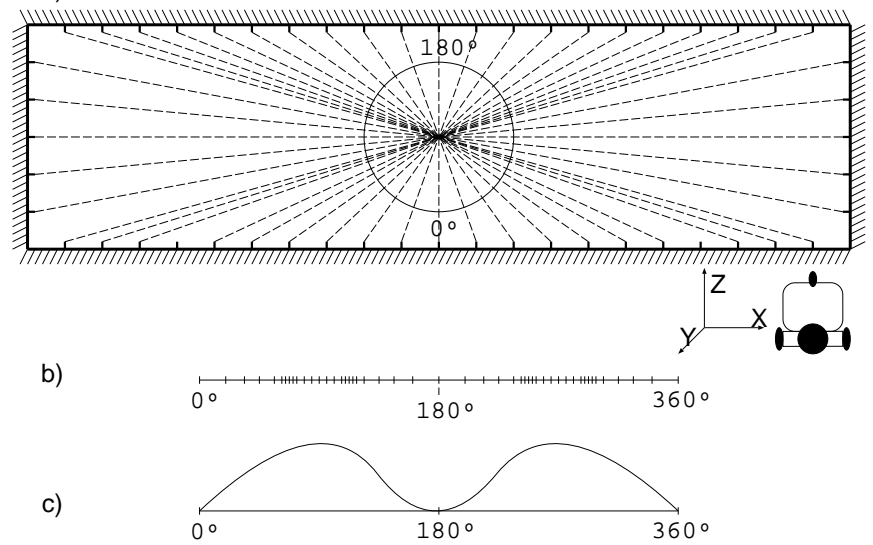

Fig. 3. a) Plane representation ( $\mathrm{X}$ and $\mathrm{Z}$ axis) of a corridor's equidistant features reflected on the hyperbolic mirror. b) Rectification of the mirror's reflection image, from $0^{\circ}$ to $360^{\circ}$. There are two differentiated zones on the surface of the mirror, where the features are more dense. These areas tend to correspond to distant objects. c) Entropy of the reflected features.

each 1-D image $\vec{l}=\left\{l_{1}, \cdots, l_{w}\right\}$ is approximated using the Shannon's discrete entropy expression:

$$
h_{i}=-\sum_{j=1}^{B} p\left(l_{j}\right) \log _{2} p\left(l_{j}\right)
$$

In this expression $B$ is the number of bins in the grayscale histogram calculated over an entropy window of width $w_{h}$ and center in $i$. This histogram is used for obtaining the probability $p\left(l_{j}\right)$ :

$$
p\left(l_{j}\right)=\frac{1}{w_{h}} \sum_{k=i-\left\lfloor w_{h} / 2\right\rfloor}^{i+\left\lceil w_{h} / 2\right\rceil} \delta\left(l_{j}-l_{k}\right)
$$

where

$$
\delta(x)= \begin{cases}1 & \text { if } x=0 \\ 0 & \text { otherwise }\end{cases}
$$

In our experiments the entropy window width is $w_{h}=21$, being $w=600$ the size of the 1-D image. We use $B=256$ 


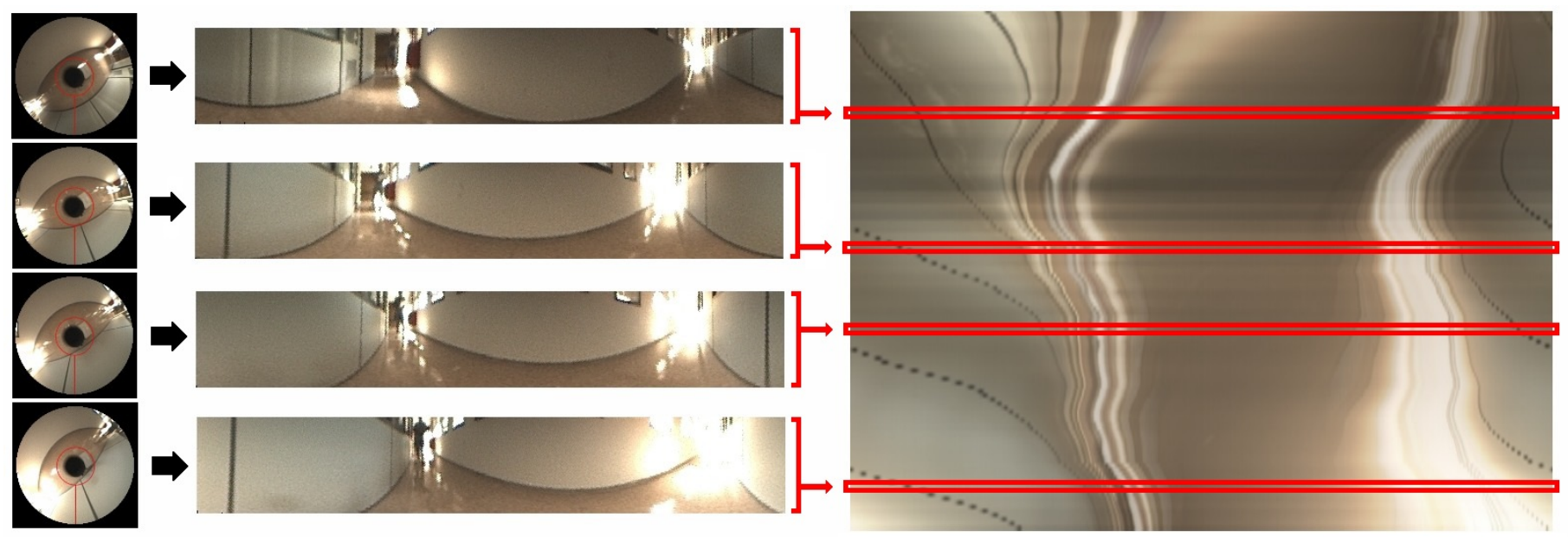

Fig. 4. The construction of the 1-D panorama images stack from omnidirectional images.
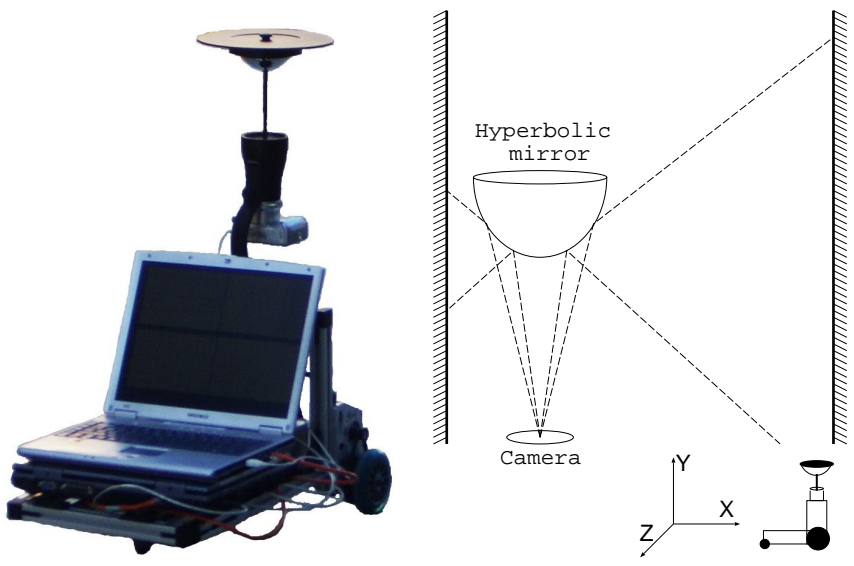

Fig. 5. Left) Catadioptric cameras installed on the PowerBot and ER-1 mobile robots. Right) The hyperbolic mirror is in vertical position (along the $\mathrm{Y}$ axis), oriented to the floor. In this way the field of vision of the camera grows vertically as the distance from the mirror increases.

bins for the grayscale histogram. In the cases for which $l_{j}$ is outside the limits of $\vec{l}$ we use $l \bmod (j, w)$, as the image is periodic and we want the entropy function to have this property too.

In Fig. 6 we represent the entropy of the sequence of 1D images of Fig. 4 which were obtained during an arbitrary indoor walk of the robot. Two lighter areas which correspond to higher entropy are well defined, and their positions in the array $\vec{l}$ correspond to angles which point to the beginning and the end of the corridor. The dark areas correspond to the walls which are near to the robot and yield lower local entropy.

\section{Fourier Series Approximation}

Two evident peaks can be observed in the entropy maps we obtain. However for their localization we have to eliminate local maxima of the curve.

This can be done by approximating the shape of the entropy function. We apply a low-pass filter in the frequency domain, keeping only the first two frequencies in the inverse transform step. In the case of two frequencies only, the Discrete Fourier

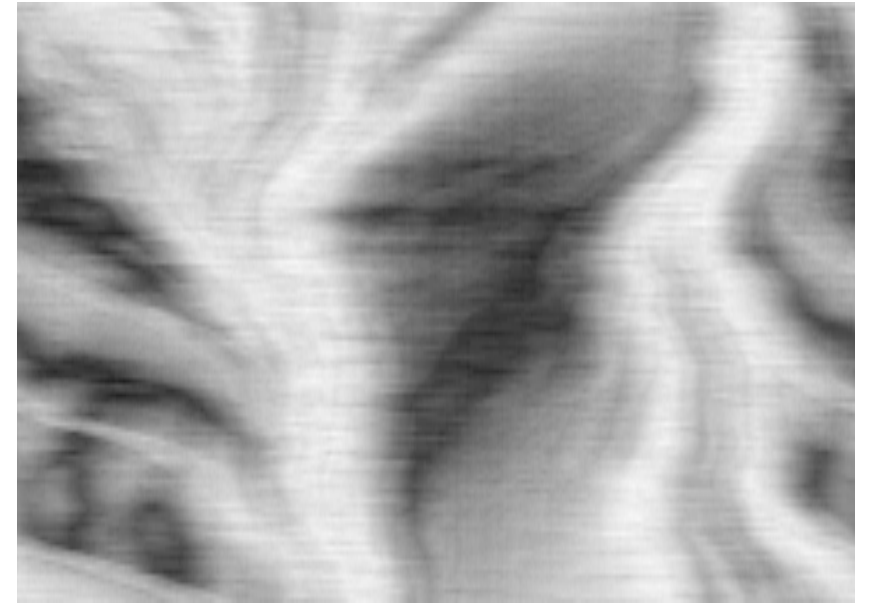

Fig. 6. The entropy map of the 1-D panorama's stack from Fig. 4. Dark tones correspond to low entropy and light tones correspond to high entropy. Image width $w=600$, entropy window size $w_{h}=21$.

Transform can be expressed as follows:

$$
\begin{aligned}
& a_{k}=\frac{1}{m} \sum_{j=0}^{2 m-1} y_{j} \cos k x_{j}, k=0,1,2 \\
& b_{1}=\frac{1}{m} \sum_{j=0}^{2 m-1} y_{j} \cos x_{j}
\end{aligned}
$$

where $m=\lfloor w / 2\rfloor$ is half the width of the 1D image. Once the principal frequencies are obtained, the inverse transform (back to the space domain) can be obtained with the following expression:

$$
S_{2}(x)=\frac{a_{0}}{2}+a_{1} \cos x+b_{1} \sin x+a_{2} \cos 2 x
$$

After this process the entropy map of Fig. 6 is transformed into the approximation of Fig. 8 where finding the maxima is trivial. The time to process one image depends on the width of the image. For a 600 pixels width it takes about $50 \mathrm{~ms}$ on a standard laptop. 


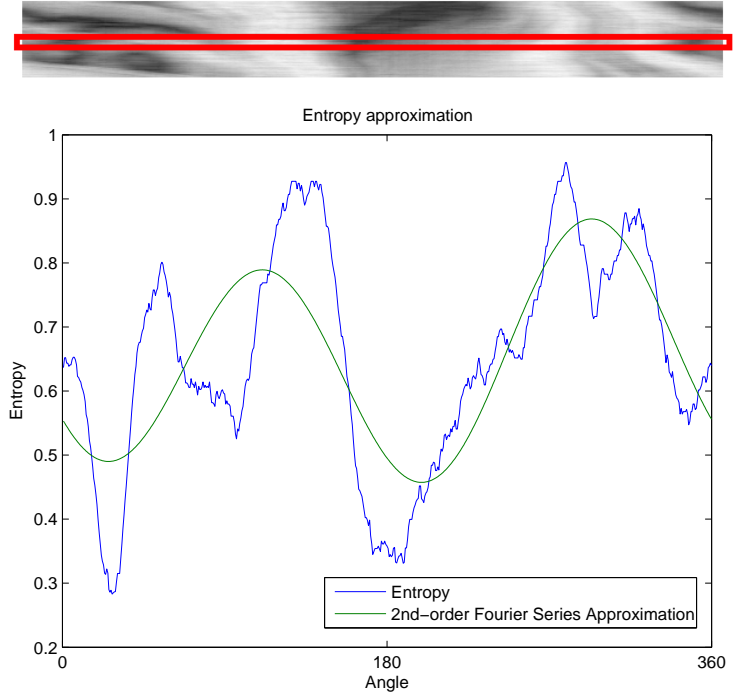

Fig. 7. Top) A line of the entropy map. It corresponds to a single snapshot of the omnidirectional camera. The first pixel corresponds to the angle $0^{\circ}$ and the last one to $360^{\circ}$. Bottom) The entropy of the marked line and its Fourier series approximation of 2 nd order, as we look for a maximum of 2 peaks in the entropy function.

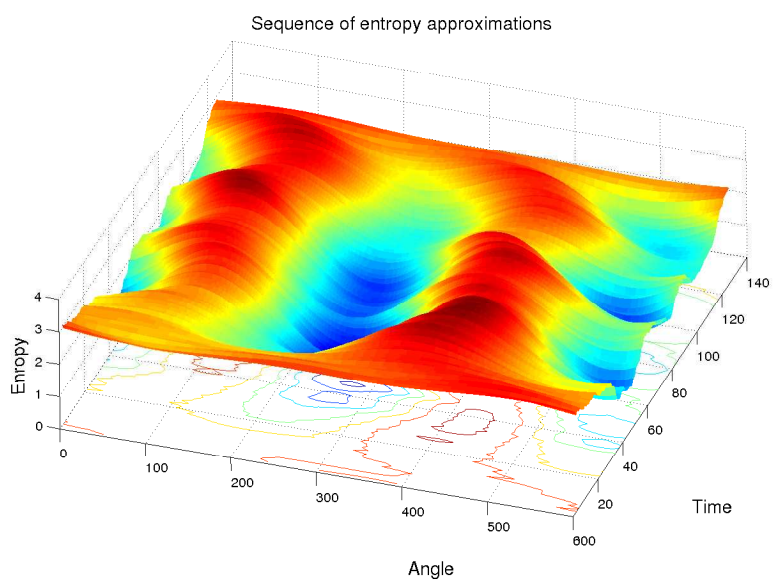

Fig. 8. A sequence of 140 entropy approximations corresponding to the entropy map represented in Fig. 6. The directions of the corridor correspond to the hills of the surface.

\section{OMNIVISUAL SONARS}

The entropy-based direction estimation is not enough for making a robot navigate safely. In Fig. 10 and Fig. 11 it can be seen that the estimated angle is not always correct. Sometimes it would mislead the robot toward a window, stairs, or another obstacle. We propose a vision-based method inspired in sonar rings, and refer to it as OmniVisual sonars (OV-sonars). OVsonars consist of virtual rays (similarly to [7]) which are launched radially from the center of the omnidirectional image and reach the first point where the gradient of the image is high (more than 0.1 in our experiments). Each $k$-th OV-sonar $\vec{V}_{k}=\left\{v_{1}, \cdots, v_{r}\right\}$ explores a maximum of $r$ pixel of the omnidirectional image. Each pixel $v_{i}$ corresponds to:

$$
\begin{aligned}
& v_{i}=I_{O}\left(i \sin \alpha+c_{x}, i \cos \alpha+c_{y}\right) \\
& \alpha=k \cdot 2 \pi / N_{s}
\end{aligned}
$$

where $I_{O}$ is the original 2D omnidirectional image with radius $r$ and center in $\left(c_{x}, c_{y}\right)$ and $N_{s}$ is the number of OV-sonars launched for each image. In our implementation we also perform a horizontal and vertical sub-pixel interpolation for smoothing the visual information.

The $\lambda(\vec{V})$ function stands for the number of pixels that the OV-sonar $\vec{V}$ explores before the $\Delta v_{\max }$ threshold is exceeded:

$$
\lambda(\vec{V})=\underset{i}{\arg \min }\left|v_{i}-v_{i-1}\right|>\Delta v_{\max }
$$

a)

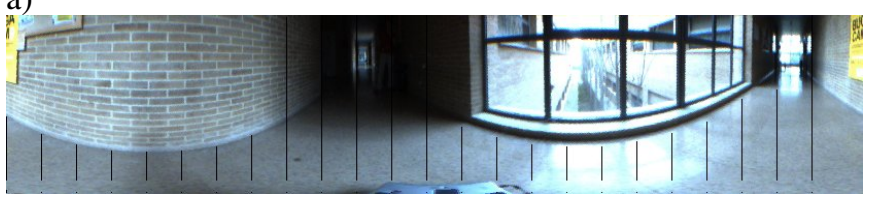

b)

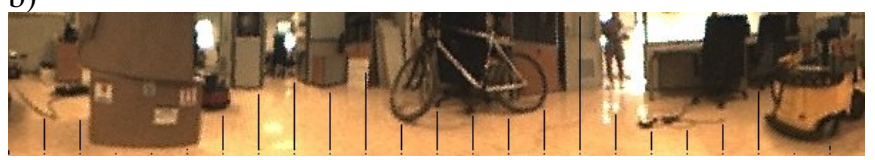

c)

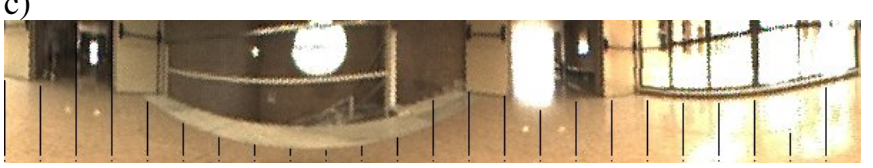

d)

Fig. 9. OmniVisual sonars with gradient threshold on different images. On a) we can see some false negatives in the dark zones of the image. On c) there is a false positive due to saturation. Note that the picture c) was taken near to stairs and a handrail which are a true positive obstacle in the example, due to the detection of a border on the floor.

The gradient measure returns a very high value when there is a strong light reflection on the image. This usually happens on gleaming surfaces with the presence of light sources and is a very common situation in indoor environments. Visually the light reflection looks like an obstacle. To solve this problem we have decided to detect light reflections and ignore them. We consider that a pixel belongs to a light reflection when in the HSV color space its saturation (S) is low and its value (V) is high. See an example on Fig. 12. The gradient of pixels with high V-S difference do not have to be considered as an obstacle. Therefore we weight the gradient $\left|v_{i}-v_{i-1}\right|$ of the expression 8 by multiplying it by the following weight function $w(i)$ :

$$
w(i)=e^{-\gamma \cdot\left(V(i)-S(i)-\frac{1}{2}\right)}
$$

where $V(i)$ and $S(i)$ are the value and saturation of the pixel $i$ in the HSV color space. The constant $\gamma$ is a value equal or greater than 1 , it regulates the sensitivity of $w(i)$ to the $\mathrm{V}-\mathrm{S}$ difference. In Fig. 13 are plotted the V-S difference and the original and weighted gradients of a OV-sonar which crosses the light reflection of the image presented in the example. In the example we used $\gamma=3$. It can be observed that after weighting the gradient function, the peaks with a large $\mathrm{V}$ $\mathrm{S}$ difference become lower, while the other become higher. Besides the $\gamma$ constant for regulating the sensitivity to light reflections, the $\mathrm{V}-\mathrm{S}$ difference can be morphologically dilated 

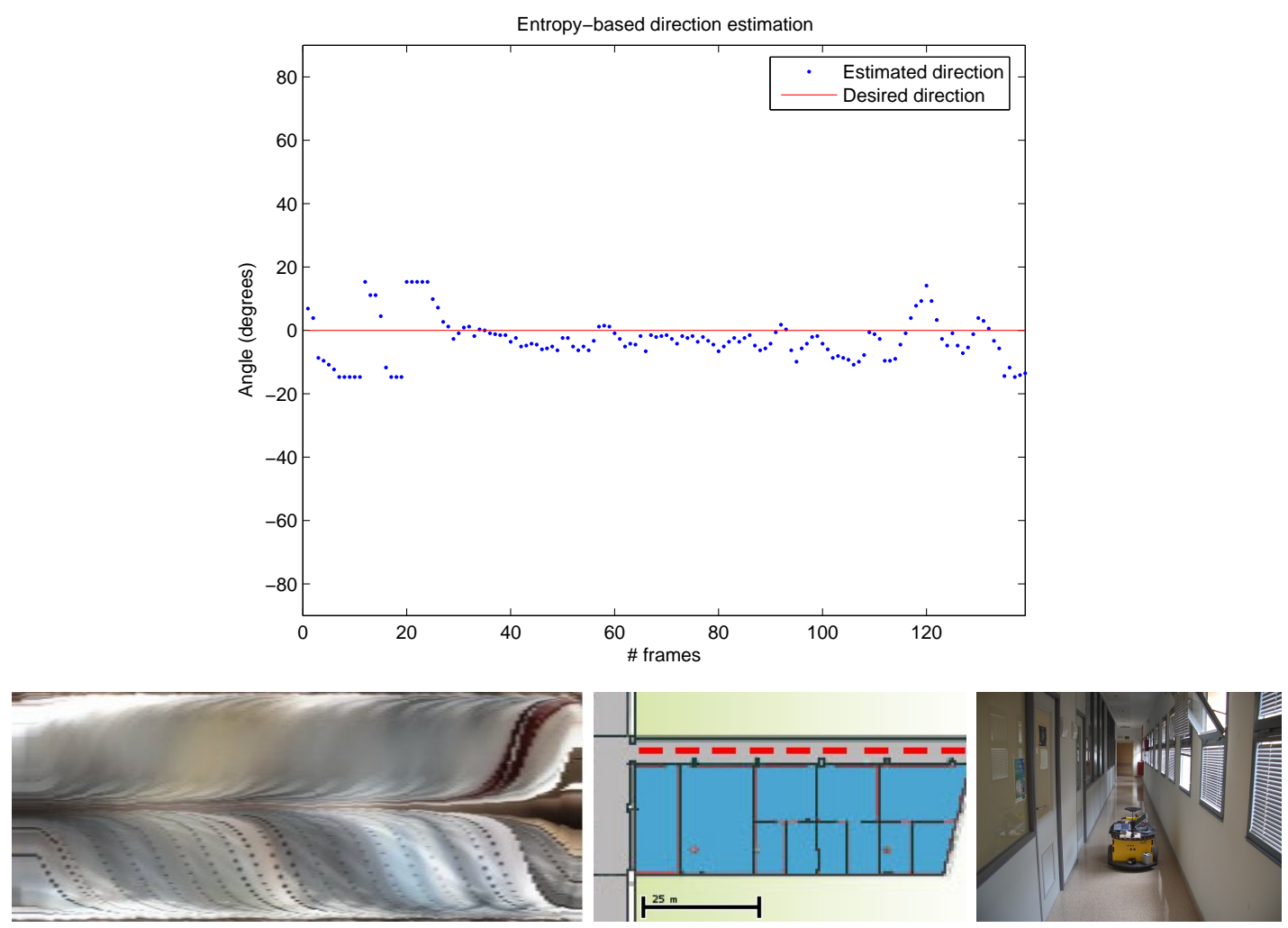

Fig. 10. Top: Results of the direction estimation test for a corridor. The estimated angle is near to $0^{\circ}$, with a mean absolute error of $5,94^{\circ}$. Bottom, left: the entropy map obtained during the experiment. Bottom, center: the straight trajectory along the corridor is represented with a dashed line. Bottom, right: a photograph of the corridor.
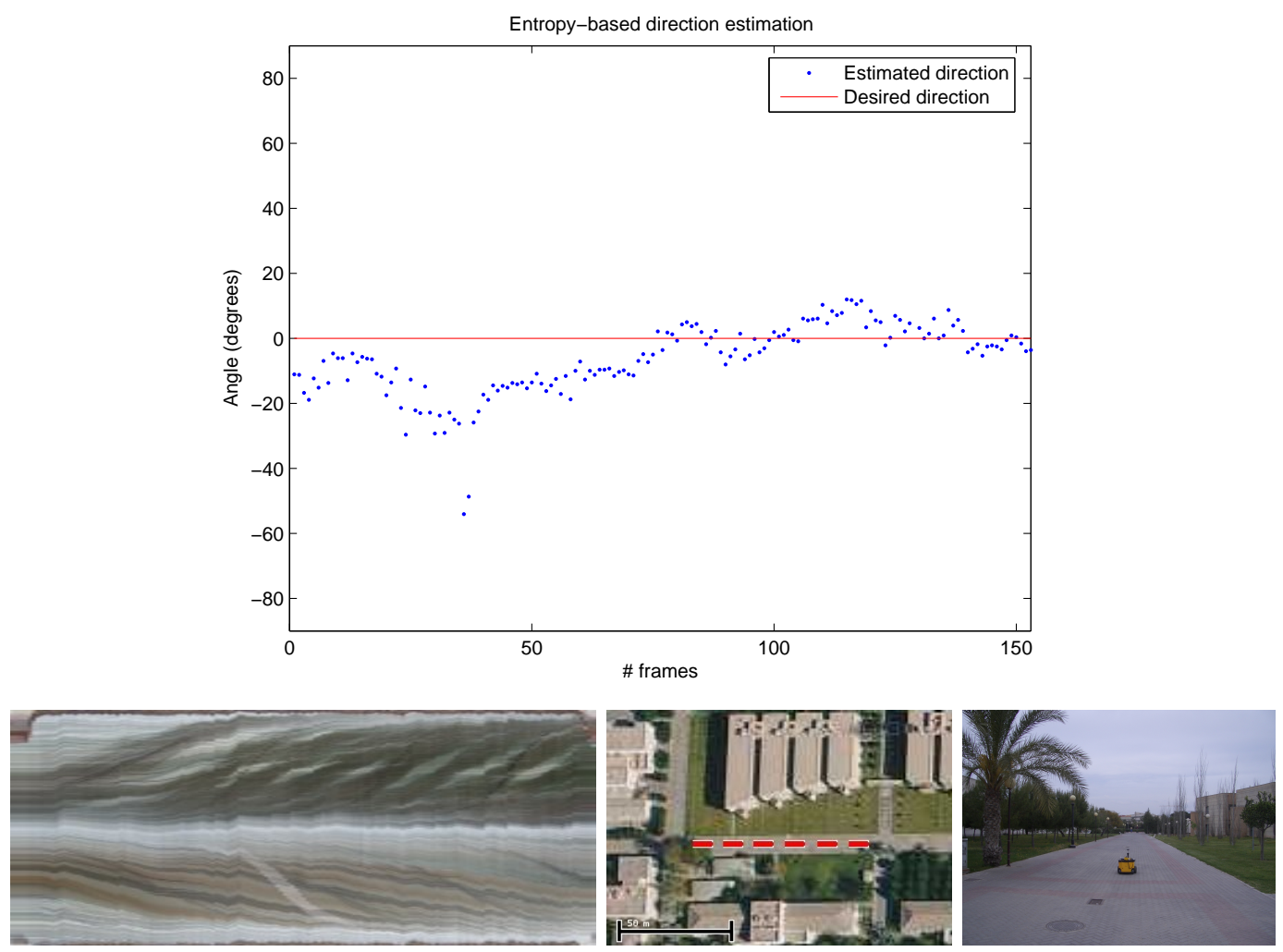

Fig. 11. Top: Results of the direction estimation test for an avenue. The estimated angle varies between $-20^{\circ}$ and $20^{\circ}$, with a mean absolute error of $10^{\circ}$. Bottom, left: the entropy map obtained during the experiment. Bottom, center: the straight trajectory along the avenue is represented with a dashed line. Bottom, right: a photograph of the avenue. 
in order to affect more the pixels which are close to a light reflection. However this operation involves additional computational cost and we do not use it in the present experiments. In Fig. 13 (bottom) we can see the final result of the OVsonars approach. The OV-sonar studied in the example does not stop at the light reflection even though there is a very strong gradient. However it stops near to the border with the wall, where the gradient is not so strong, but there is a lower probability of light reflection.
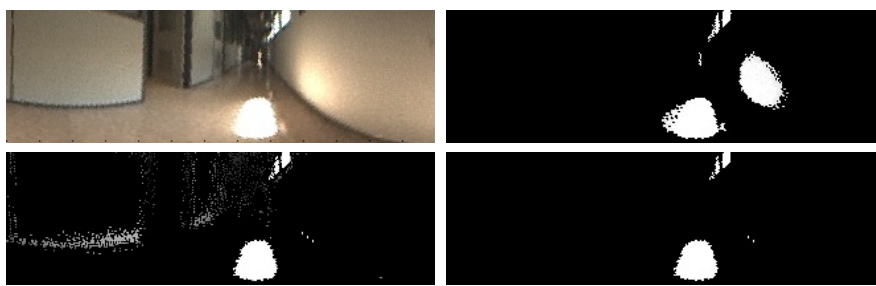

Fig. 12. Localization of light reflections. Top left: Image presenting a strong light reflection on the floor. Top right: pixels which have a high $\mathrm{V}$ (value) in the HSV color space. Bottom left: pixels which have a low S (saturation) in the HSV space. Bottom right: intersection of high $\mathrm{V}$ and low $\mathrm{S}$ pixels.

Gradient is a poor measure for highly textured floors, and we are working on improving the OV-sonars technique. However it works in many corridors, as camera's limited resolution softens the texture. Another problem is the need of adjusting the gradient threshold $\Delta v_{\max }$ and the light reflection sensitivity $\gamma$, as these parameters are not the same for all environments. In Fig. 9 we present three examples of OV-sonars in different environments. In our experiments we launch $24 \mathrm{OV}$-sonars per frame and it takes less than $50 \mathrm{~ms}$ to process them. This computational time depends on the height of the image (100 pixels in our experiments) and the number of OV-sonars. A larger number of these would only help for the detection of small obstacles while increasing the computational load.

$\mathrm{OV}$-sonars detect obstacles and free zones in the environment. The following section details how this information is used for navigation.

\section{NAVIGATION}

Behavior-based architectures have proved to be convenient for systems which have various objectives and environmental restrictions simultaneously [8]. In the previous sections we presented two basic behaviors, one of them for guiding the robot, and the other one for avoiding collisions. Following we propose the combination of both of them in the same system.

A simple navigation method for robots with a sonar ring [11], or OV-sonars in our vision-based system,is the sum of force vectors:

$$
\begin{aligned}
\vec{f}_{k} & =d_{\max } \cdot \widehat{v}_{k}-\vec{v}_{k} \\
\vec{f}^{*} & =\sum_{k=1}^{N_{s}} \vec{f}_{k}
\end{aligned}
$$

where $N_{s}$ is the number of $\mathrm{OV}$-sonars and $\vec{f}^{*}$ is the resulting vector whose direction would avoid the collision. We set $d_{\max }=r$ the radius of the omnidirectional image (see Fig. 16).
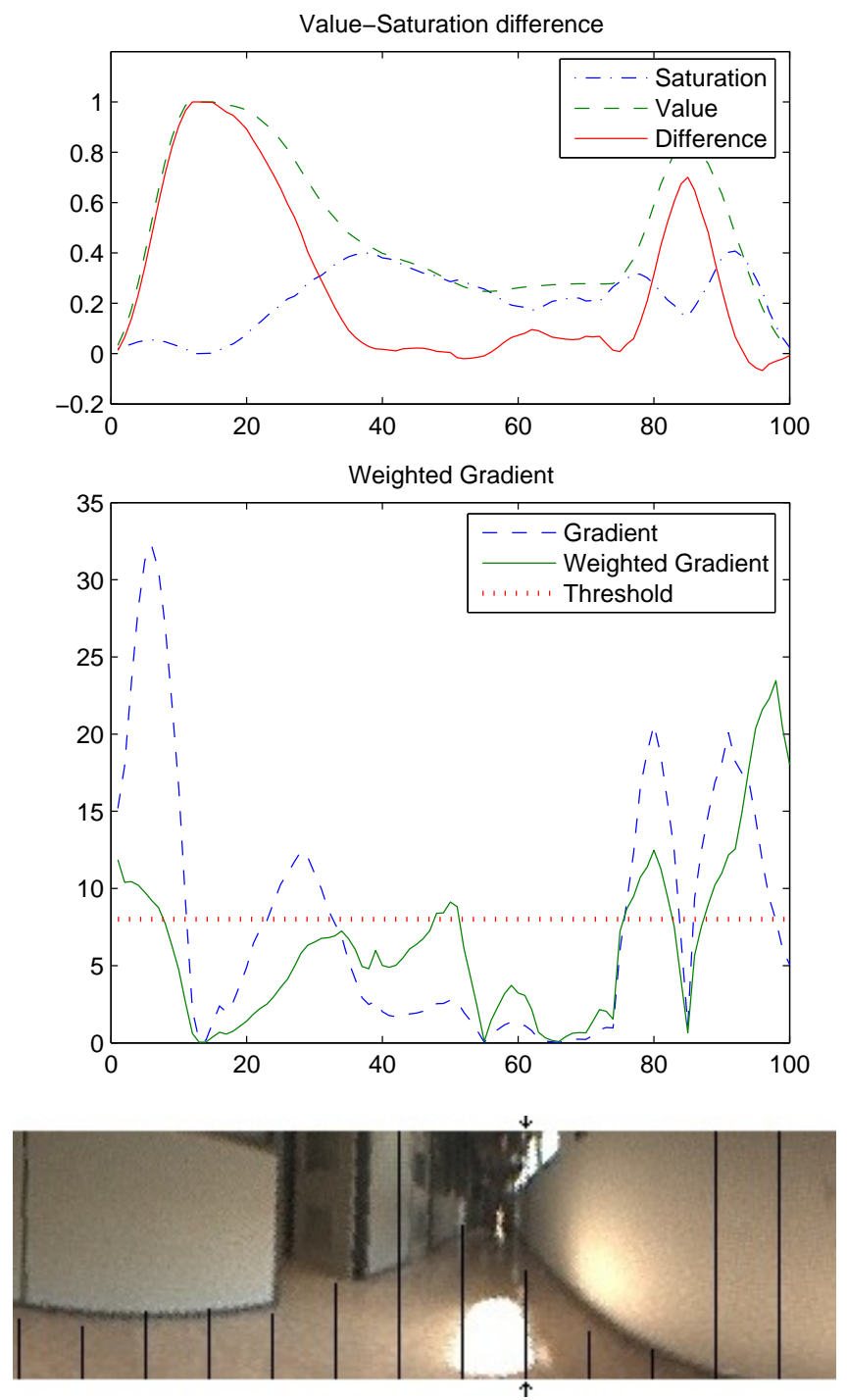

Fig. 13. Top: difference between Value and Saturation for the pixels of a single OV-sonar. Center: the gradient of the same OV-sonar and its weighting according to the V-S difference. Bottom: The OV-sonars on the image. The $\mathrm{OV}$-sonar used in the previous plots is marked with arrows.

Our implementation relies on the entropy-based estimation of the direction for guiding the robot whenever OV-sonars yield magnitudes larger than the safety distance $d_{\text {safe }}$ (measured in pixels). If it is invaded, the robot's direction and speed go under the control of the direction and magnitude of $\vec{f}^{*}$. See Algorithm 1. In our application the overall process of capturing a frame, estimating the direction, calculating the OV-sonars, and sending the speed commands to the robot takes less than $200 \mathrm{~ms}$ on a standard laptop with a $600 \times 100$ resolution in the rectified panorama images.

\section{EXPERIMENTAL SETUP}

The experiments we present in this paper were performed with an ActivMedia PowerBot robot and the Player-Stage library. We have successfully run the navigation behaviors on a Magellan Pro robot with Player-Stage, and on an Evolution Robotics ER-1 robot, using the manufacturer's API. The omnidirectional mirror is the Remote Reality's OneShot360. 


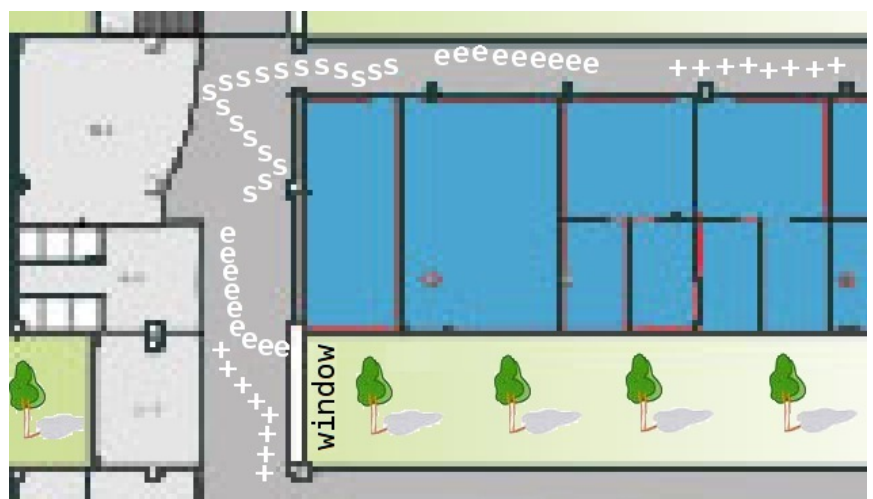

Fig. 14. Some of the trajectories described by the robot while navigating indoors. a) The 'e' signs stand for navigation based only on entropy. b) The 's' signs stand for OmniVisual sonar (OV-sonar) based navigation. c) The ' + ' signs stand for the combined behavior which used both entropy and OmniVisual sonars.

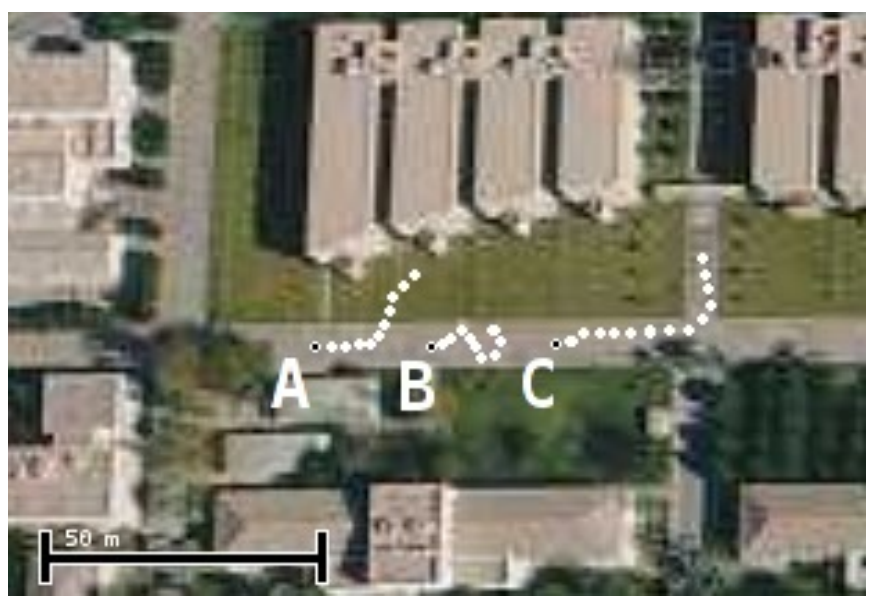

Fig. 15. Some of the trajectories described by the robot while navigating outdoors. a) Trajectory described while guided only by entropy. b) Trajectory yielded by OmniVisual sonars (OV-sonars). c) Combined behavior.

We started using a low resolution Logitech webcam, and for the present experiments we installed a GreyPoint Flea2 firewire camera.

The first experiment tests the entropy-based behavior presented in section II. We forced the robot to follow a straight trajectory along a) a corridor (indoor) and b) an avenue (outdoor). In Fig. 10 and 11 the results of the direction estimation are plotted. The indoor estimation is better than the outdoor one.

When the robot is guided by the estimated direction values, its position keeps on changing, and so do the estimations. In some cases the robot is misguided to some object, and when approaches it, the view changes and the trajectory is corrected. In other cases the robot crashes, for example when it approaches a large window and the outside's entropy is much higher than indoors. Some of the trajectories obtained during the experiments are represented in Fig. 14 and 15. Three kinds of trajectories are represented: guided by the estimated direction, guided by OV-sonars, and guided by both of them.

On the one hand the entropy estimation yields a trajectory that is better oriented than using only OV-sonars. On the

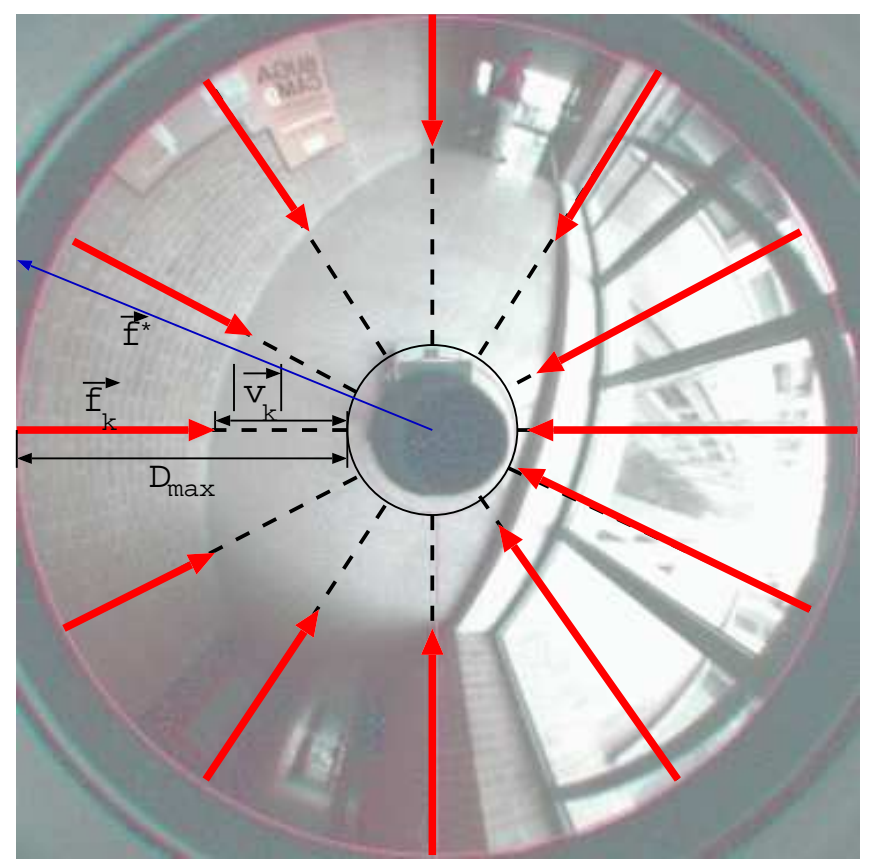

Fig. 16. The force vectors $\vec{f}_{k}$ (red thick arrows) represent the difference between the maximum distance $d_{\max }$ and the vectors of the OV-sonars $\vec{v}_{k}$ (dashed lines). The sum of all force vectors $\vec{f}^{*}$ is represented with a blue thin arrow.

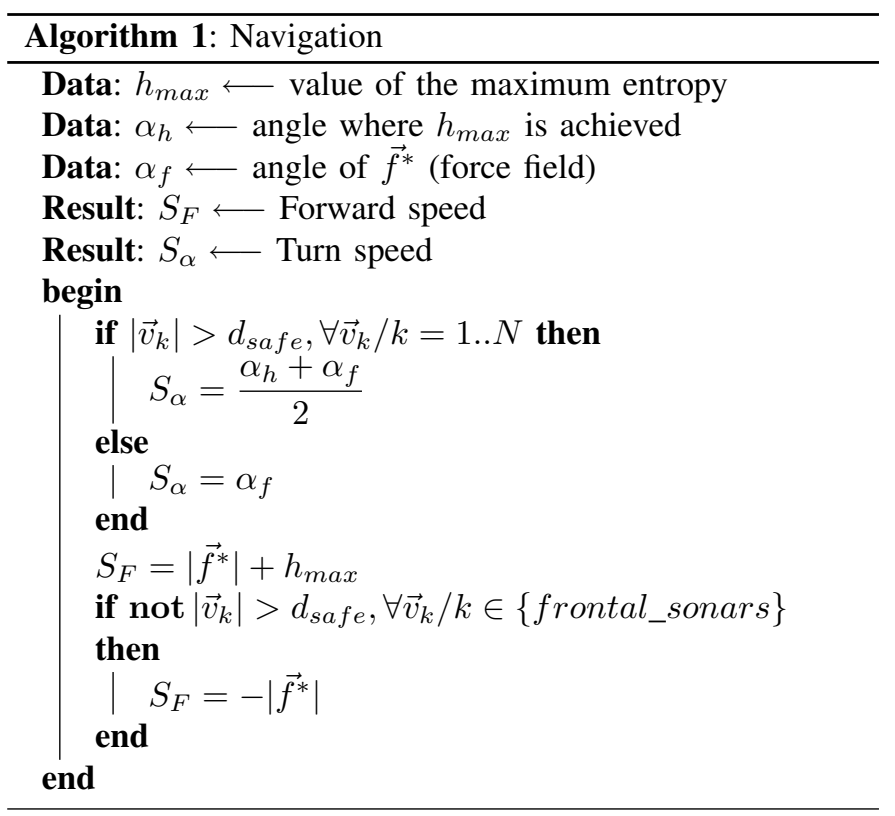

other hand it does not keep a safety distance from walls and obstacles, and it crashes into the window (in the indoor tests, Fig. 14) shortly after the outside trees come into the vision field. OV-sonars avoid such crashes and enable the robot to cross doors and even navigate along a corridor. The combined behavior keeps the safety of OV-sonars while entropy provides the robot with a better heading angle in most parts of the indoor environment.

Regarding the outdoor tests we can see that in the entropyguided trajectory the robot is mislead by some entropy peak at the left, and it goes over the grass (Fig. 15.a). The tra- 


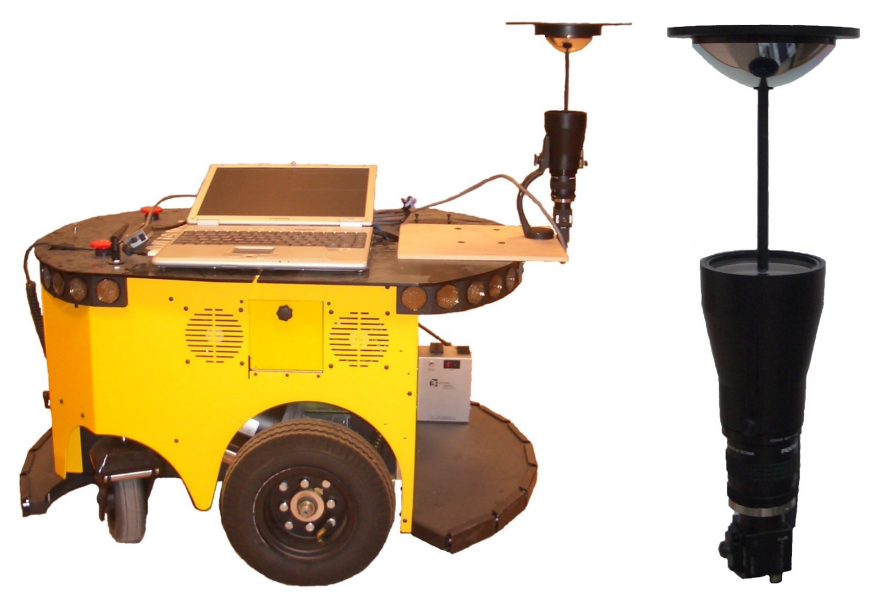

Fig. 17. ActivMedia PowerBot mobile robot and a Remote Reality omnidirectional mirror and a PointGrey Flea2 firewire camera. (The sonar ring of the robot is not used in any part of the current work).

jectory yielded by OmniVisual sonars (OV-sonars) uses no information about direction, however it keeps the robot on the surface of the avenue. There are also some irregularities due to shadows, sewer covers and other features. Finally the combined behavior keeps the robot from going into the grass, while providing the angle of a hypothetical direction.

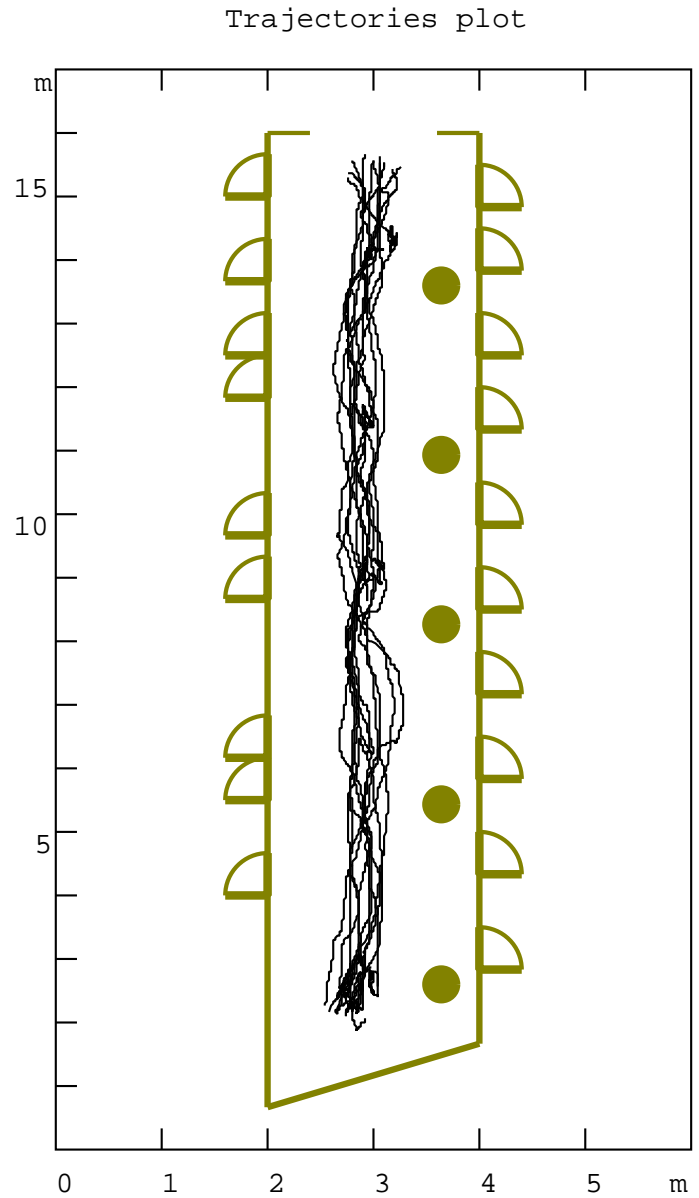

Fig. 18. Plot of ten different trajectories performed by the robot under similar conditions.
In Fig.18 ten different trajectories are plotted. The starting point is the same and the robot manages to get to the other end of the corridor in eight of them. In the other two cases it gets stuck in local minima. This is due to some reflections on the floor which make the obstacle avoidance trap the robot in an infinite cycle. This is a current limitation of the obstacle avoidance behavior, as it is purely reactive and has no mechanism for getting out of local minima. Another drawback that can be observed is the variability from one experiment to another.

A video recording of the omnidirectional-vision-based navigation is available on-line [17].

\section{CONCLUSIONS AND FURTHER WORK}

In this work we have developed two basic behaviors the combination of which allows a robot to navigate along different structured environments. The first behavior is entropybased and estimates the direction of the corridor while the other behavior is inspired in sonars and keeps the robot away from obstacles.

These behaviors rely only on omnidirectional vision. This is an advantage as a catadioptric sensor can easily be installed on any mobile robot. The method works in a variety of environments without a priori knowledge and without any learning process. Any omnidirectional camera can be used without the need of calibration, as the method is non-metric. Also, it has a very low computational cost.

The disadvantage of vision-based approaches is that vision is not as reliable as range sensors like sonars or lasers. However we have showed examples in which vision performs better, for example with the presence of very low obstacles like grass. On the other hand if a robot is equipped with range sensors they can also be used for safety, in case vision fails.

A drawback of this vision-based approach is that the OVsonars method we use is very simple. We are currently working on improving it so it can work for any kind of surface. We intend to perform a multi-scale entropy analysis of the pixels which the OV-sonar crosses. A significant change in the multiscale entropy space would mean a change in the texture, independently of its size. We are also considering the use of Jensen-Rényi divergence measure for texture segmentation.

Another future work is to improve the sonar-based navigation algorithm and compose it with the entropy-based direction estimator via fuzzy logic. It is also necessary to implement a mechanism for getting out of the local minima. Finally, we plan to use these behaviors in a vision-based topological navigation system.

\section{ACKNOWLEDGMENT}

This research is funded by the project DPI2005-01280 from the Spanish Government and the project GV06/134 from Generalitat Valenciana (Spain).

\section{REFERENCES}

[1] A. Briggs, C. Detweiler, Y. Li, P. Mullen, and D. Scharstein, Matching scale-space features in $1 D$ panoramas. Computer Vision and Image Understanding, 103(3):184-195, September 2006 
[2] D. Lambrinos, R. Möller, T. Labhart, R. Pfeifer and R. Wehner, A mobile robot employing insect strategies for navigation. Robotics and Autonomous Systems, special issue on Biomimetic Robots, Vol. 30:3964, 2000

[3] C. Geyer and K. Daniilidis, Structure and motion from uncalibrated catadioptric views. In IEEE Conf. Computer Vision and Pattern Recognition, Hawaii, Dec. 11-13, 2001

[4] Y. Yagi, K. Imai, K. Tsuji and M. Yachida, Iconic Memory-Based Omnidirectional Route Panorama Navigation. IEEE Transactions on Pattern Analysis and Machine Intelligence, Vol. 27, No. 1, January 2005

[5] J. Gaspar and J. Santos-Victor, Vision-based Navigation and Environmental Representations with an Omni-directional Camera. IEEE Transactions on Robotics and Automation, Vol. 16, No. 6, December 2000

[6] C. Lopez-Franco, E. Bayro-Corrochano, Unified Model for Omnidirectional Vision Using the Conformal Geometric Algebra Framework, 17th International Conference on Pattern Recognition (ICPR'04), Vol. 4, pp. 48-51, 2004

[7] S. Lenser and M. Veloso, Visual sonar: Fast Obstacle Avoidance using Monocular Vision, In Proc. of the IEEE/RSJ IROS 2003

[8] R.C. Arkin, Behavior-based robotics. The MIT Press, ISBN 0-26201165-4, 1999

[9] N.Y. Ko, R.G. Simmons, Teh Lane-Curvature Method for Local Obstacle Avoidance. IEEE/RSJ International Conference on Intelligent Robots and
Systems, Canada, October 1998

[10] J. Borenstein and Y. Koren, Real-time Obstacle Avoidance for Fast Mobile Robots. IEEE Transactions on Systems, Man, and Cybernetics, Vol. 19, No. 5, pp. 1179-1187, September/October 1989

[11] O. Khatib. Real-time obstacle avoidance for manipulators and mobile robots. The International Journal of Robotics Research, 5(1), 1986.

[12] S. Benhimane and E. Malis, A new approach to vision-based robot control with omni-directional cameras. In proc. of the 2006 IEEE International Conference on Robotics and Automation, Orlando, May 2006

[13] G.L. Mariottini, D. Prattichizzo and G. Oriolo, Image-based Visual Servoing for Nonholonomic Mobile Robots with Central Catadioptric Camera. In proc. of the 2006 IEEE International Conference on Robotics and Automation, Orlando, May 2006

[14] M. Cover and J.A. Thomas, Elements of Information Theory. Ed. Wiley Interscience, ISBN: 0-471-24195-4, 1991

[15] J.M. Sáez, M.A. Cazorla and R. Rizo, Métodos Matemáticos en la Computacion, Ed. Universidad de Alicante, 2002

[16] J.D. Faires and R. Burden, Numerical Methods. Ed. Brooks Cole, 3rd edition, June 2002

[17] B. Bonev, M.A. Cazorla and F. Escolano, Robot Navigation Video, http://www.dccia.ua.es/ boyan/navigation.avi, 2007 